\title{
Die Nibelungen und anderes Volk - Fahrten in eine fremde, unheimliche Welt ${ }^{1}$
}

\section{Kathryn Smits}

In seiner kleinen historischen Schrift zur Tausendjahrfeier Österreichs schreibt Herwig Wolfram:

[...es] verlaufen Grenzen von großer Beständigkeit innerhalb Österreichs; sie umfangen es nicht, sie sind ihm eingeschlossen. In Krisenzeiten werden diese Nahtstellen deutlich erkennbar. Wer über die Enns nach Osten ging, kam einst zu den 'Hunnen'. Ob nun zum Volk Attilas oder zu den ihm nachbenannten Awaren und Ungarn, der Reisende zog wie Kriemhild und die Nibelungen in eine fremde, unheimliche [...] Welt. Umgekehrt ist es aber nicht anders. Für die Pannonier begrenzt die Enns das Land der Menschen, und westlich von ihr beginnen Chaos und Verlassenheit. Bis dahin kamen ihre Reiter, ohne sich zu verlieren. Mit ihrer Überschreitung wurde die Enns [...] nur zu oft ein Fluß ohne Wiederkehr. ${ }^{2}$

Um diese Nahtstelle - die Enns im metaphorischen Sinne - geht es hier: nicht nur im Nibelungenlied, sondern auch in anderem Zusammenhang. Um Grenzen also zwischen Heimat und ellende (wie es im Mittelalter heißt), zwischen Menschen und Menschen, vor weit mehr als 1000 Jahren und in unserer eigenen Zeit.

Das mhd Lexem ellende bedeutet 'außer Lande' und ist natürlich die

${ }^{1}$ Dieser Vortrag (hier leicht revidiert) richtete sich an eine Hörerschaft, die sich größtenteils aus Neugermanisten, aber auch aus allgemein Interessierten ohne spezielle Kenntnisse der mhd Literatur zusammensetzte. Beim Zitieren der mhd Fachliteratur beschränke ich mich auf das Nötigste. Schwer verständliche mhd Zitate wurden übersetzt.

${ }^{2}$ Herwig Wolfram: 1000 Jahre und mehr. Österreich und seine Menschen um das Jahr 1000, (Österreich Dokumentation), Bundespressedienst, Wien 1996, S. 30. 
Wurzel des heutigen Begriffs 'Elend' bzw. 'elend'. ${ }^{3}$ Wer die Heimat verließ, stand in früheren Jahrhunderten (und steht in weiten Teilen der Welt auch heute noch) außerhalb des Schutzes der Rechtsgemeinschaft, in die er hineingeboren wurde, und damit gleichsam außerhalb der menschlichen Ordnung. Was einem solchen Menschen begegnet ist aventiure, will sagen: das intensive Erleben einer verschärften Situation, das zur Steigerung aller Lebenskräfte führt. Für den, der die aventiure sucht (im Gegensatz zu dem, der flieht oder von der Gemeinschaft ausgestoßen wird), ist sie das, was seinem Leben erst Sinn verleiht. Diese gewollte Konfrontation mit dem höchsten Risiko ist auch ein Movens zum Aufbruch der Nibelungen ins Land der Hunnen, trotz der Schicksalhaftigkeit ihres Unterfangens. Die êre (mhd heißt das die 'äußere Ehre', der Ruhm, die Anerkennung durch andere) gilt ja auch über den Tod hinaus. Das mag in dem von Fatalismus durchtränkten Erzählstoff des Nibelungenliedes nicht sonderlich betont sein, Dichter und Publikum aber wußten sehr wohl darum und sorgten - gerade durch den Vorgang des Erzählens, durch Niederschrift und Zuhören - gemeinsam für diese weitere Dimension. Mhd aventiure bedeutet ja auch "Zeugnis von einem Abenteuer": Erlebnis und Bericht sind in diesem Wort begrifflich verschmolzen. Erst die Literarisierung verleiht letzten Endes der aventiure ihren Sinn. ${ }^{4}$

${ }^{3}$ Jacob Grimm bezeugt seine Freude am Lexem elend und dessen Etymologie: "urbedeutung dieses schönen, vom heimweh eingegebnen wortes ist das wohnen im ausland, in der fremde, und das lat. exsul, exsilium, gleichsam extra solum stehen ihm nahe." Deutsches Wörterbuch von Jacob und Wilhelm Grimm, Leipzig 1862, Bd. 3, Sp. 406ff.

${ }^{4} \mathrm{Im}$ folgenden verwende ich - bewußt eigenwillig und auf andere 'Abenteuer'-Situationen ausgeweitet - das mhd Lexem aventiure, weil es semantisch mehr beinhaltet und einen größeren Spielraum bietet als der heutige Begriff 'Abenteuer'. Zur Definition von aventiure im Sinne der Artusepik schreibt Ehrismann: "Mit aventiure verbanden sich [...] die Merkmale «+Ritter», «+Waffen», «+Suche», «+Kampf», «+Ruhm», $\ll+$ (gesellschaftliches) Ansehen». Es war eine freiwillige, auch dem 'Zufall' überlassene Konfrontation mit den Gefahren und Herausforderungen der Welt, einem 'Zufall', der im Rahmen des mittelalterlichen Weltbildes auch von Gott gesteuert sein konnte [...]. Es war keine militärische Leistung im Auftrag eines Herrn, wenn sie manchmal auch im Rahmen einer militärischen Aktion vollzogen wurde [...]." Otfrid Ehrismann: Ehre und Mut, Aventiure und Minne. Höfische Wortgeschichten aus dem Mittelalter, München 1995, S. 23. Daneben wird aventiure in der Bedeutung 'Vorlage', 
Die Sehnsucht nach aventiure war nur allzuoft mit bitterer Notwendigkeit gepaart. Sichtbar wird das zum Beispiel in einem altkurdischen Lied aus dem achten vorchristlichen Jahrhundert:

Da die Quellen versiegt, das letzte Grün des Grases verbrannt, die zerrissene Krume verdorrten Bodens, des Ackers fruchtbare Decke als Staub durch den Gluthauch des Windes emporgewirbelt Wolken bildet, spürst du die Ohnmacht, siehst das verendende Tier, hörst das Weinen der Kinder, und deine Fäuste schaffen nicht Wasser, geben den Gräsern nicht Leben, schützen nicht die fruchtbare Erde des Ackers vor dem Gluthauch des Windes Zweifelst du? schreit dein Herz nicht in Zorn vor dem Tag : Vergeblich duckt sich das Tier, geduldig das Sterben erwartend, Du aber erhebe dich! $!^{5}$

Getrieben durch dieses Amalgam aus Not und Abenteuerlust brachen die Menschen immer wieder ins Ungewisse auf. Die Vikinger taten es, die Seefahrer und 'boat people' aller Zeiten, die Auswanderer, die im neunzehnten Jahrhundert unter großen Entbehrungen mit Kind und Kegel in Segelschiffen nach Neuseeland und Australien kamen, - und die Flüchtlinge und Aussiedler jeder Art, die sich heutzutage von Osten her kommend in den Städten Westeuropas einfinden, nicht zuletzt in Österreich.

Wenn ich mich hier in Dunedin mit diesem Thema befasse und dabei das

'Quelle', 'Dichtwerk', 'Kapitel' verwendet. Der Begriff ist schillernd, nicht leicht definierbar und wird in der Helden- und Artusdichtung auch unterschiedlich verwendet.

${ }^{5}$ Zitiert nach: Carola Meier-Seethaler: Ursprünge und Befreiungen. Die sexistischen Wurzeln der Kultur, Frankfurt a.M. 1992, S. 254. 
Nibelungenlied zentral stelle, so möchte ich vorweg die ersten Besiedler Neuseelands erwähnen. Ihre Seefahrten nämlich, ihre aventiuren, waren die kühnsten, navigatorisch gekonntesten, die es in der Weltgeschichte je gegeben hat. Diese Fahrten sind genauso von Sagen umwoben wie die Züge der Völkerwanderung Europas. Zwischen dem dritten und zwölften Jahrhundert besiedelten die Polynesier die weitverstreuten Inseln des gewaltigen Pazifischen Ozeans, darunter Neuseeland. ${ }^{6}$ Freilich fanden sie hier nur eine Tier- und Pflanzenwelt vor und wurden deshalb nicht zu Fremden in diesem Land, sondern - als dessen erste menschliche Bewohner - zu den tangata whenua, wie es in der Maori-Sprache heißt, zu den 'Kindern des Landes'. So kommt es, daß die Frage nach Fremdsein und Heimischsein hierzulande anders gestellt werden muß als in Europa. Das freilich ist ein Thema, mit dem wir uns hier nicht beschäftigen werden. Bleiben wir trotzdem noch einen Moment bei den Neuseeländern. In unseren Tagen treibt die Sehnsucht nach Abenteuer und Risiko die Menschen nämlich nicht nur in den Weltraum, was nur einer kleinen Zahl von Astronauten vergönnt ist, sondern auch $\mathrm{zu}$ dem in diesem Land erfundenen Bungi-Jumping, wobei der Abenteurer freilich von einem Seil gesichert ist: Angst am Gummiband also, ohne nennenswertes Risiko, für die man die Kreditkarte hinhält (ein Erlebnis, daß sich nicht wesentlich von dem des Kindes meiner Schweizer Freundin unterscheidet, das mit rotem Kopf von der Achterbahn stieg und atemlos sagte: "So viel Angst für einen Franken!"). Auf der Suche nach der 'verschärften Situation' meint der im Wohlstand lebende Mensch, diese nur noch in künstlich erzeugten Streßsituationen finden zu können. Im Spiel mit der Todesangst hofft man das aventiure-Erlebnis nachzuvollziehen. Für die im Nibelungenlied dargestellten Männer freilich war diese Fahrt in die

${ }^{6}$ James Belich: Making Peoples. A History of the New Zealanders from Polynesian Settlement to the End of the Nineteenth Century, Auckland/London/New York 1996, S. 16: "When Europeans discovered just how many islands [the Polynesians had settled], they were stunned. How could stone-age folk have spread themselves from Hawai'i in the north, on the Tropic of Cancer, to Stewart Island in the south, just short of the subantarctic; from Tonga in the west to Easter Island in the east, traversing the great Pacific like a garden pond many centuries before Europeans made it across the petty Atlantic? Some scholars felt forced to posit a giant sunken Pacific continent, of which the Polynesian Islands were remnants left high and dry, but most confronted the facts: here were the greatest voyagers the world had ever seen." 
Fremde blutiger Ernst: ein ihnen auferlegtes Schicksal, dem sie nicht entrinnen konnten und das sie mit Würde zu tragen verstanden.

Erinnern wir uns, worum es im Nibelungenlied geht. Es wurde bald nach 1200 von einem anonymen Österreicher gedichtet, spielt aber viel früher, in der Zeit der Völkerwanderung. Allerhand gerade noch sichtbare historische Ereignisse und ein breites, mündlich überliefertes Sagengut sind darin verflochten. Interessant ist, $\mathrm{da} ß$ der in diesem Epos geschilderte Untergang des Burgundenstammes in Wirklichkeit nicht mit einer heroisch-abenteuerlichen Reise ins ferne Hunnenland, an den Hof des Hunnenfürsten Attila verbunden war. Soweit überhaupt nachweisbar beruht die Handlung auf folgendem historischen Geschehen. Der Stamm der Burgunden hatte sich, kommend aus Richtung Borkholm, um das Jahr 400 in der Gegend von Worms niedergelassen. In der nächsten Generation vermehrte er sich dort stark. Im Jahr 437 suchten die Burgunden dann ihr Gebiet nach Westen hin (also nicht in Richtung Hunnenland) zu vergrößern. In anderen Worten: Sie erhoben sich, genötigt durch Hunger, genau wie die kurdischen Hirten im Lied, und zogen ins linksrheinische Belgica. Daran jedoch wollten die Römer - in dieser letzten Phase vor dem Kollaps ihres großen Reiches - sie hindern, und es war ein von den Römern gedungenes Hunnenheer, das dann hier, westlich vom Rhein, den Stamm der Burgunden dezimierte. (Was von ihnen übrigblieb, wurde bald darauf von den Römern im heutigen Burgund angesiedelt.) Der geschichtliche Attila war an der Niederlage der Burgunden in Belgica nicht beteiligt. Er fiel erst 451 in die linksrheinische Region ein. ${ }^{7}$

Das Nibelungenlied (und vor ihm das germanische Sagengut, aus dem es schöpft) deutet das historische Motiv der Landsuche also ins Abenteuerlich-Heroische um. Es wird berichtet, wie die drei Burgundenkönige, die Brüder Gunther, Gernot und Giselher, ihren Sitz in Worms haben. Siegfried (er stammt aus einem separaten, nordischen Sagenkreis) erhält Gunthers Schwester Kriemhild zur Frau. Vorher hat Siegfried mittels seiner Tarnkappe - dem Gunther geholfen, die sagenhaft starke

${ }^{7}$ Werner Hoffmann: Das Nibelungenlied, (Sammlung Metzler 7), Stuttgart 61992, S. 40ff.; Otfrid Ehrismann: Nibelungenlied. Epoche-Werk-Wirkung, München 1987, S. 62ff.; Hermann Reichert: Nibelungenlied und Nibelungensage, Wien/Köln 1985, S. 118ff.; Lutz Mackensen: Die Nibelungen. Sage, Geschichte, ihr Lied und sein Dichter, Stuttgart 1984, S. $44 \mathrm{ff}$. 
Brunhild als Braut zu gewinnen. Als später der dabei an Brunhild verübte Betrug ruchbar zu werden droht und Siegfried den Burgunden politisch gefährlich geworden ist, plant man seinen Mord. Umgebracht wird er vom vornehmsten Gefolgsmann und Ratgeber der Burgundenkönige, Hagen von Tronege, dem abgründigsten, härtesten aller männlichen Gestalten des Nibelungenliedes. Der Witwe Kriemhild raubt Hagen das von Siegfried ererbte Vermögen, den sogenannten Nibelungenhort, damit ihr die Mittel zur Rache fehlen. Jahre später, da außer Hagen kein Mensch mehr an Kriemhilds Rache glaubt, stimmt diese einer Ehe mit Etzel zu. Etzel: das ist der schon erwähnte Hunnenkönig Attila, dessen Residenz Gran sich weit weg im Osten befindet, wahrscheinlich circa 20 Kilometer nördlich vom heutigen Budapest. ${ }^{8}$ Nach Jahren der Ehe in der Fremde lädt nun Kriemhild ihre Brüder zu sich ein. Sie weiß, daß ihr Widersacher Hagen seine Dienstherren zwangsläufig begleiten muß. Die Brüder, zunächst recht ahnungslos, ziehen daraufhin gen Osten, freilich mit einem großen, bewaffneten Heer, denn dazu rät Hagen. Als dann beim Überqueren der Donau märchenhafte Wasserfrauen dem Hagen bestätigen, was dieser ohnehin schon ahnte: daß nämlich das Heer bis auf den letzten Mann in den Tod gehen wird, zerstört Hagen das einzige Boot, damit keiner seinem Schicksal entfliehen kann. Dann aber steht er bedingungslos zu seinen Dienstherren und seinen Kameraden (Strophen 1581-1590). ${ }^{9}$

Uns Heutige mutet das gewiß als eine perverse Auffassung von Treue an. Vergessen wir aber nicht, daß sie noch weit in unser zwanzigstes Jahrhundert hinein als exemplarisch galt. In beiden Weltkriegen wurde auf beiden Seiten von den jungen - oft noch minderjährigen und nicht wahlberechtigten - Soldaten eine Vaterlandstreue verlangt, die uns heute mehr als bedenklich erscheinen mag.

Ziehen wir noch eine Parallele. Im Mai 1996 begab sich ein anderer Trupp auf aventiure. Er überquerte die bewußte Nahtstelle. Sie lag diesmal nicht in Österreich, hieß nicht Enns, sondern es handelte sich um den Mount Everest. Der Widersacher war der Berg selbst mit seinen extremen Wetterverhältnissen. Wo sonst findet der heutige Zeitgenosse, der um jeden Preis etwas Extremes erleben will, denn noch die totale aventiure?

${ }^{8}$ Hoffmann, S. 43f.; Mackensen, S. 53ff.; Ehrismann, S. 308.

${ }^{9} \mathrm{Karl}$ Bartsch, Helmut de Boor, Roswitha Wisniewski (Hg.): Das Nibelungenlied, Wiesbaden, ${ }^{22} 1988$. 
$\mathrm{Da} ß$ übrigens gerade der Mount Everest, dieses gewaltige Naturphänomen, den heutigen Bergsteigern durchaus, vermenschlichend, als Gegner im nibelungschen Sinne gilt, das bezeugt der Kommentar eines der beiden Erstbesteiger, des Neuseeländers Sir Edmund Hillary, der 1952 nach vollbrachter Tat befriedigt feststellte: "We knocked the bastard off".

Um Everest-Touristik handelt es sich also hier, um den höchsten Berg der Welt statt Attilas und seiner Hunnen. Für den Veranstalter ist das ein lukrativer, wenn auch riskanter Beruf. Die Teilnehmer - es sind Gleichgesinnte aus aller Welt mit meist reicher Bergsteigererfahrung - findet er übers Internet. Es ist in unseren Tagen nicht mehr die Treue zu einem Gefolgsherrn, die die Mannen treibt, sich dem Reiseführer anzuvertrauen, sondern - wie es heutigem Usus, heutigen Möglichkeiten entspricht - der individuelle Drang zur 'verschärften Situation'. Wie Hagens Weggefährten, hatte diese Everest-Bruderschaft sich die Sache etwas kosten lassen. ${ }^{10}$ Der eine Reiseführer zum Everestgipfel hieß Rob Hall. Er war Neuseeländer und einer der erfahrensten Bergsteiger unserer Zeit. Im Vordergrund stand bei Hall wohl weniger das Geschäft als der unwiderstehliche Drang zur aventiure. Er starb für dieselbe Ehrauffassung, die Hagen in Bezug auf seine Stammesgenossen an den Tag legte. Als einer von Halls Kunden nach erfolgreicher Besteigung, hoch oben, gerade unterhalb des Gipfels, nicht weiter konnte, ließ dieser ihn nicht allein. Der Kunde starb, und Hall selbst wußte sich im zunehmend schlechten Wetter verloren. ${ }^{11}$

Hagen von Tronege und Rob Hall trennen Jahrhunderte - und eine Welt. Der auffallendste Unterschied ist wohl, daß der Bergsteiger die aventiure

${ }^{10}$ In Bezug auf das frühe Mittelalters heißt es bei Herwig Wolfram, S. 14: "Die Ausrüstung des vollbewaffneten Kriegers bestand [...] aus dem Helm, dem doppelt so teuren Panzerhemd, der Brünne, ferner aus Schild, Lanze, Schwert, Kurzschwert, Pfeil und Bogen sowie einem Pferd. Wollte der Krieger dazu auch noch Beinschienen anlegen, so mußte er noch einmal soviel zahlen wie für einen Helm. Schild und Lanze machten davon bloß ein Drittel aus. Die Vollbewaffnung kostete enorme Summen und stellte den Gegenwert von ganzen Rinderherden oder einem Dutzend Höfen dar."

${ }^{11}$ Risiko und Dilemma eines solchen Bergführers hat Reinhold Messner formuliert. Dieser hatte sich, ebenfalls im Mai 1996, geweigert einen großzügig zahlenden Amerikaner zum Everestgipfel zu begleiten. "Meine Antwort war, daß ich im Notfall die Sicherheit des Gastes nicht gewährleisten, daß ich mich vielleicht nur selber retten kann. Deshalb habe ich abgelehnt." Der Spiegel 37, 9.9.1996, S. 128. 
nicht mehr im blutigen Kampf gegen die als fremd, feindselig und ethisch minderwertig eingeschätzte Kreatur sucht, ob es sich dabei nun, in verschiedenen Jahrhunderten, in Mythen oder Realität, um Hunnen, Riesen, Drachen, Andersgläubige, Indianer, Safari-Löwen oder um Wale handelte. Weiter trennt diese beiden Männer ihre unterschiedliche Einstellung zum anderen Geschlecht und damit zur Gesellschaft überhaupt. Können wir uns einen Hagen von Tronege vorstellen, dessen letzte Gedanken der daheimgebliebenen Frau und dem ungeborenen Kind gegolten hätten? Wir brauchen uns gar nicht erst vorzustellen, daß er, wie es der moderne Rob Hall tat, per Handy in die ferne Heimat telefoniert, um die letzten Augenblicke mit seiner schwangeren Frau zu teilen. Daß Hagen in der Vorstellungswelt des Dichters verheiratet ist, müssen wir wohl annehmen, denn welcher Mann seines Standes hätte sich damals nicht um Nachkommen bemüht? Hagens Welt aber wird vom Dichter als eine Männerwelt per se dargestellt, und auch Trost findet er am Ende ausschließlich bei den Kameraden. Rob Halls Lage war anders. Seine Frau war in Sachen aventiure seine gleichgestellte Partnerin: sie ist Ärztin und hat als bewährte Bergsteigerin auch selbst schon den Everestgipfel erreicht. Es tut sich also ein wesentlicher Unterschied zwischen unserer Zeit und jener auf, in der die Frauen entweder passiv klagend daheimzubleiben hatten oder aber, sobald sie sich wie Kriemhild in die Domäne der Männer vorwagten, in deren Augen zu Denaturierten werden mußten. Denn im Nibelungenlied, gedichtet um 1200, wird durchaus nicht die Realität der um Jahrhunderte zurückliegenden Völkerwanderung dargestellt, in der Frauen und Männer notgedrungen gemeinsam in die fremde, unheimliche Welt zu ziehen hatten. Es sind in diesem vom Schicksal geprägten Heldenepos die Rollen der Geschlechter paradoxerweise nach höfisch-literarischem Protokoll getrennt, nicht zuletzt auch nach dem im zwölften Jahrhundert stark hervortretenden theologischen Prinzip, daß dem Mann im Leben die aktive, der Frau die passive Rolle gebühre.

Trotz dieser Unterschiede ist unverkennbar, daß in einem Zeitgenossen wie Rob Hall etwas weiterlebt, was auch die erschreckende Hagengestalt prägt. In diesen beiden Gestalten verschränken sich aventiure und der Trieb, das Leben bzw. den Tod bis aufs äußerste herauszufordern. Rob Hall hatte schon vier erfolgreiche Everestbesteigungen hinter sich. Er wußte, genausogut wie Hagen von Tronege, daß sein Gegner ein Killer war, wenn nicht heute, so doch morgen. Ein dunkler Trieb hieß ihn 
trotzdem dorthinzugehen. Genau betrachtet ist ja auch die Lage von Halls Frau nur im Detail eine andere als die der Nibelungenwitwen. Es wird das uralte Muster sichtbar: Die Männer sterben heroisch, die Frauen leben weiter und haben gefälligst die Verantwortung für den Nachwuchs zu tragen. Drittens ist ja auch Rob Hall, nur wenige Monate nach seinem Tod, schon zum - umstrittenen - Helden einer Erzählung, einer aventiure in der zweiten Bedeutung dieses Wortes geworden. Diese Literarisierung hat in den Medien stattgefunden, nicht nur in seiner Heimat Neuseeland, sondern in 'Newsweek', dem 'Spiegel', 'Vogue', der Reisezeitschrift 'Outside' und weltweit in zahllosen anderen Publikationen. ${ }^{12}$ Auch darin gleicht er Hagen von Tronege.

Die Einstellung dieser beiden Recken, Hagen und Hall, unterscheidet sich nun aber in einem wesentlichen Punkt von der der Ritter in den Artusromanen, die - auf dem Höhepunkt der mhd Literatur um 1200 - fast gleichzeitig mit dem Nibelungenlied entstanden. Den aventiuren dieser Ritter wohnt ein anderer Sinn inne. Die Wende des zwölften zum dreizehnten Jahrhundert brachte ein neues ethisches Prinzip ins Spiel. Nicht der heldenhafte Tod war jetzt gefragt. Nicht er war das Ziel Erecs, Iweins, Gawans. Diese Ritter der Artusepik gewinnen nach Punkten, lehren den Gegner zumeist buchstäblich Mores, indem sie sein Leben schonen und ihm das ethische Verhalten à la König Artus nahebringen. Sie sind, wenn man so will, Missionare der höfischen $z u h t$, sind auf ein Ziel ausgerichtet, das zwar ungeheuere Courage, nicht aber den Tod erfordert, weder den eigenen, noch den des Gegners. Diese höfisch-literarische Tradition, die mit der Wirklichkeit des damaligen Ritteralltags nur wenig gemein hatte, ${ }^{13}$ färbt bis heute auf unser sittliches Denken ab. Daneben blieb aber die Nibelungenmentalität weiterbestehen. In den Recken des Nibelungenliedes lebt etwas fort, das uns Heutige in seiner Radikalität erschreckt und abstößt, uns andererseits in dieser wunderbaren literarischen Gestaltung auch zutiefst fasziniert. Diese ambivalente Leserreaktion teilen wir übrigens mit dem Dichter. Man spürt zuweilen deutlich, daß ihm seine Gestalten nicht ganz geheuer sind. Eine seiner großartigsten Leistungen ist es aber, daß er sich nicht dazu hat verleiten lassen, das ${ }^{12}$ Der Spiegel 37, 9.9.1996, S. 126.

13 Joachim Bumke: Studien zum Ritterbegriff im 12. und 13. Jahrhundert, Heidelberg 1964, S. 130-148, bes. S. $138 \mathrm{ff}$. 
Erzählte moralisch zu werten. Zwar ändert er manches ins Höfische ab, er führt uns aber auch ein Verhalten vor Augen, das zeitlos ist, das sich in unserer eigenen Kultur in abgewandelten Formen wiederfindet. Fast ungeschminkt begegnen wir im Nibelungenlied, vor allem in der Gestalt Hagens, den Geist einer Völkerwandergesellschaft, für welche Stammesfeindschaft, Krieg und Totschlag eine Selbstverständlichkeit waren. $\mathrm{Daß}$ sich dieses Verhaltensmuster, trotz der Bildung und Kultur vieler Jahrhunderte, in Europa wie auch anderswo immer wieder regt, das zeigt nicht nur die Geschichte der beiden Weltkriege, sondern auch das Geschehen unserer eigenen Tage, dieser Epoche einer neuen Völkerwanderung: in Bosnien, in Srilanka, wie auch in Ruanda und Liberien. Wie viele Kinder wachsen heute heran, deren entscheidende Jahre von Mord, $\mathrm{Haß}$ und Gewalt geprägt sind, deren Wertskala - wie die Psychologen ahnen - für immer den Stempel dieser Erfahrungen tragen wird, so daß sie diese Lebenshaltung tradieren und ihre Kinder und Kindeskinder unweigerlich auch wieder von diesem Trauma geprägt sein werden? Übrigens: Was hat das Kind Hagen gesehen, daß es zum Mann wurde, der er ist? ${ }^{14}$ Interessanterweise gibt uns der Nibelungendichter auf diese Frage tatsächlich eine Antwort: Hagen von Tronege habe seine Jugend an Etzels Hof verbracht, heißt es, als Geisel bei den Hunnen (Str. 1752-1757). Das hieß damals allerdings etwas anderes als heute. Der Junge war dort von seiner Sippe zwar unfreiwillig zurückgelassen bzw. hingeschickt worden, als Garant des guten Willens, er wurde aber an diesem Hof nicht anders erzogen als jeder andere Edelknabe auch. Der Dichter also will durchaus nicht sagen, daß Hagen bei den Hunnen eine minderwertige bzw. eine besonders grausame Erziehung genossen hätte. Im Gegenteil. Er schildert Etzels Hof in diesem Zusammenhang höchst positiv. Die Darstellung der Hunnen im Nibelungenlied ist (darauf kommen wir noch zurïck) nicht konsequent. Braucht der Dichter sie als Widersacher seiner heldenhaften Burgunden, so werden sie um des Kontrastes Willen negativ geschildert.

${ }^{14}$ Wir sollten nicht vergessen, daß die Gestalten des Nibelungenliedes nicht als Charaktere im neuzeitlichen Sinne zu verstehen sind, sondern daß ihnen, wie auch den Figuren anderer mhd. Dichtungen, eine andere künstlerische Auffassung zugrunde liegt. So ist auch Hagen nicht als psychologisch konsequent dargestellte Person zu verstehen, sondern eher als Figur, die eine bestimmte Rolle innehat. Dieser Rückblick auf 'seine' Kindheit dient also nicht dazu, Hagen psychologisch zu deuten, sondern ist eher ein Versuch, dem von ihm vertretenen Männertypus nahezukommen. 
Etzels Hunnenhof dagegen wird durchweg als exemplarisch dargestellt, Etzel selbst als Muster eines friedlichen höfischen Herrschers. (Etzels Passivität ist im Sagenkreis um Dietrich von Bern vorgezeichnet, erinnert andererseits aber auch an Artus.) Die hövescheit in der mhd Literatur bildet - das gilt auch für den Nibelungendichter - ein Netz, das die ganze in diesen Dichtungen geschilderte Welt umspannt, die als Einheit empfundene Christenheit, aber auch - soweit damals bekannt - die Welt der Andersgläubigen. Natürlich hat es nach dieser Vorstellung auch Herrscher gegeben, die böse handelten, indem sie diese höfische Norm verletzten bzw. verneinten (Etzel gehörte nicht zu ihnen), aber eine andere Norm gab es nicht. Wahres Kriegertum, Unerschrockenheit und List hat das Kind Hagen also am Hunnenhof gelernt, es ist dort zum musterhaften Ritter erzogen worden. In der Hagengestalt wird das dem Nibelungenlied zugrundeliegende Paradox, diese Spannung zwischen dem höfischen Geist der Literatur um 1200 und der Lebenspraxis der germanischen Recken, besonders deutlich sichtbar. Dieser Mann, der gemäß seiner Erziehung doch eigentlich ein höfischer Kosmopolit hätte werden sollen, erweist sich als Haudegen alten Schlags, dessen bedingungslose Treue zur eigenen Sippe und zu den eigenen Dienstherren jede Versöhnlichkeit ausschließt. Dieser Hagen von Tronege also steht nicht als fürs Leben Geschädigter da, sondern als beispielhafter Recke. Gerade das ist das Faszinierende und zugleich das Erschreckende: daß in der Welt, die uns hier trotz der höfischen Bemühungen des Dichters vor Augen geführt wird, die Verrohung eines jeden Mannes als Tugend galt. Sogar als unerläßlich galt sie und als selbstverständlich. Der Nibelungendichter wertet nicht, sondern schöpft aus dem Sagenquell und stellt nahezu kommentarlos dar. Immer stoßen wir auf diese - künstlerisch so wirkungsvolle - Ambivalenz des Dichters in Bezug auf seinen Stoff.

In der Literatur der Zeitgenossen des Nibelungendichters finden wir freilich, wie schon angedeutet, viele Spuren einer neuen, humaneren Haltung. Mehrmals sind es Frauengestalten, die ihr zum Ausdruck verhelfen. Vor allem bei Wolfram von Eschenbach. In seinem Parzival setzt die durch aventiure verwitwete Herzeloyde alles daran, ihrem verwaisten Sohn das Ritterschicksal zu ersparen. Der junge Parzival geht trotzdem, zwangsläufig, in die Männerwelt hinaus, übersteigt aber die in der Artusgesellschaft gültigen Werte souverän und verleiht der triuwe ein 
neues Gesicht. Der sensible Aspekt von Parzivals Wesen schreibt der ausgesprochen 'genetisch' denkende Dichter dem mütterlichen Erbe zu. Wolframs späteres Epos Willehalm ist, für damalige Verhältnisse, sogar ein geradezu pazifistisches Manifest. Zwar werden dort die Schlachten zwischen Christen und Andersgläubigen (Sarazenen) bis in jedes schreckliche Detail beschrieben, gerade dadurch wird aber die Erzählung transparent, so daß sie den Blick auf die Sinnlosigkeit des dargestellten Gemetzels freigibt. Es ist nicht zufällig eine an der Nahtstelle zwischen den Kulturen stehende Frau, die im Willehalm den Männern Einhalt zu gebieten sucht: die zum Christentum bekehrte Gyburc, Willehalms Gattin, deren Sippe auf der anderen Seite kämpft und deren Herz zerrissen ist. Gegen alle Männerspielregeln verstoßend mischt sie sich in die Diskussion ein und fleht die Ritter an:

und ob der heiden schumpfentiur ergê, sô tuot daz sælekeit wol stê:

hoert eins tumben wîbes rât: schônt der gotes hantgetât. $\quad(306,25-28)^{15}$

In Wolframs Werk wird sichtbar, daß die neue Ethik um 1200, natürlich stark beeinflußt durch die von Cluny ausgegangene Kirchenreform, der systematischen Verrohung der Männer die Stirn geboten hat. Überhaupt liegt die Tendenz zu einem humaneren Umgang mit der Kreatur, sei es Mensch, sei es Tier, in der Luft. Die Kreuzzugsbegeisterung ist abgeklungen. Nur wenige Jahre trennen die Entstehung des Nibelungenliedes (ca. 1204) von dem Beginn der Wirksamkeit des Franz von Assisi jenseits der Alpen (1209). Und in Österreich selbst dichtete Walther von der Vogelweide um diese Zeit:

Nieman kan mit gerten

kindes zuht beherten.

den man zêren bringen mac,

${ }^{15}$ 'Und wenn Ihr die Heiden besiegt, so versündigt Euch nicht: Hört auf den Rat einer unerfahrenen Frau, verschont sie, denn auch sie hat Gott geschaffen.' Text und Übersetzung nach: Wolfram von Eschenbach: Willehalm. Text der 6. Ausgabe von Karl Lachmann. Übersetzung und Anmerkungen von Dieter Kartschoke, Berlin 1968, Verse 306,25-28. 
dem ist ein wort als ein slac.

dem ist ein wort als ein slac,

den man zêren bringen mac.

kindes zuht beherten

nieman kan mit gerten. $\quad(\mathrm{L} 87,1-8)^{16}$

Das paränetische Thema dieses Palindroms ist nicht neu, es hat eine jahrhundertealte Tradition und läßt sich besonders in den Heiligenviten des Mittelalters nachweisen. ${ }^{17}$ Interessant ist freilich, daß dieses Prinzip gerade zu diesem Zeitpunkt auch im Rahmen der höfischen Dichtung als Gegenstand eines Walther-Liedes und gleichzeitig bei Wolfram (in der Gurnemanzlehre des Parzival, 174,7-9) auftaucht.

Beim Nibelungendichter steht die Tendenz zur Gewaltlosigkeit durchaus

16'Niemand vermag mit Worten die Erziehung eines Kindes zu erzwingen. Der, den man zu einem Mann von Ehre zu erziehen vermag, dem ist ein Wort wie ein Schlag.' Mhd Text zitiert nach Carl von Kraus, Karl Lachmann, Hugo Kuhn (Hg.): Die Gedichte Walthers von der Vogelweide, ${ }^{13} 1965$.

${ }^{17}$ Claudia Opitz: Frauenalltag im Mittelalter. Biographien des 13. und 14. Jahrhunderts (Ergebnisse der Frauenforschung 5), Weinheim 1991. Zur Behandlung der (später zu Heiligen avancierten) Kinder im Kloster, vor allem der Mädchen, heißt es hier, S. 49f.: "Auch die Erziehungsmaßnahmen Belohnung und Strafe - waren diesen besonderen Vorstellungen von der Beschaffenheit des kindlichen Körpers und der jungen Seele, die dieser beherbergt, verpflichtet. Kindheit, meinte etwa Dietrich von Apolda, sei ein Zustand, in dem der Mensch unfähig sei, Schmerz zu ertragen oder seinen Verstand zu gebrauchen. [...] Dem sollten denn auch die Erziehungsmethoden angemessen sein. Wenngleich die Strafe mit dem rüetelîn eine Forderung zumindest der kirchlichen Autoritäten war, wie sie auch Berthold von Regensburg in seinen Predigten um die Mitte des 13. Jhdts vertrat, so [sei] doch auch eine harte und unangemessene Strafe eher schädlich und sollte vermieden werden. [...] Noch deutlicher bringt dies Vinzenz von Beauvais zum Ausdruck, wenn er bemerkt, daß die Strafen durch Tugenden kontrolliert werden müßten: durch Strenge, durch Sanftmut und Vernunft, und durch Bescheidenheit. [...] Er ist dabei beileibe kein 'progressiver' Einzelgänger [...] - auch die Hagiographen gestalten ihre Texte ganz in diesem Sinn. [...] Taucht das Problem 'Strafen' in den Viten auch selten auf - die heiligen Kinder sind ja schon vorbildlich und müssen kaum erzogen werden - so sind doch einige Ausführungen heranzuziehen, die im wesentlichen eher gewaltfreie Straf- und Erziehungsmaßnahmen bekunden." Es fällt auf, daß Walther nicht nur Gewalt ablehnt, sondern auch den Rat erteilt, mit Worten an den Verstand des Kindes zu appellieren. In den weiteren Strophen seines Liedes betont er im Einklang mit Vinzenz von Beauvais die tugenden des Erziehers und die Notwendigkeit des guten Beispiels. 
nicht im Vordergrund. Seine Sache war es, über Helden alten Schlags zu berichten. Trotzdem hatte auch er an diesem neuen Denken Anteil. Im Nibelungenlied fällt in diesem Zusammenhang die Gestalt des Dietrich von Bern auf, der bewußt als friedlicher Gegenpol zum Gewaltmenschen Hagen von Tronege konzipiert sein mag. Beide sind - unter unterschiedlichen Bedingungen - im Hunnenland als Fremde zu Gast. Beide werden unter unterschiedlichen Bedingungen - stark provoziert. Der Dichter aber schildert in ihnen ein geradezu spiegelbildliches Verhalten in Bezug auf Gewalt. $^{18}$

Die 'Sagengestalt Dietrich von Bern (Bern bedeutet nicht das schweizerische Bern, sondern Verona) basiert auf dem historischen Gotenkönig Theoderich den Großen (geboren 471, gestorben 526), hat aber faktisch mit diesem Urbild so gut wie nichts mehr zu tun. In der weitverbreiteten Sagentradition ist Dietrich ein Außenseiter, der stets das Richtige weiß und tut. Auch er ist ein ellender, aber kein Dienstmann, sondern ein König, der sein Reich verloren hat. Die Sage hat ihn - unhistorisch - an Attilas Hof versetzt. Dort weilt er als Freund, nicht als Vasall. Dietrich von Bern war, schon als Sagentypus und ehe ihn der Nibelungendichter in sein Werk einbaute, zum Repräsentanten des noblen Heimatvertriebenen geworden. ${ }^{19}$

Was nun die Dietrich-Darstellung im Nibelungenlied angeht, so gibt es wohl keine Gestalt, die den Dichter thematisch, d.h. vom Sagenstoff her, so sehr einengte wie Dietrich von Bern. Durch die mündliche Sagenüberlieferung hatten die Hörer genaue Vorstellungen, was und wie dieser Dietrich zu sein hatte. Denn vom Nibelungendichter erwartete das Publikum nicht, daß er Neues erfand. Altvertrautes in neuem Gewand wollte man hören, Erzählstoff, welcher durch die lange Überlieferung den Stempel des Wahren trug. Ein Dichter, der erfand, setzte sich dem Vorwurf aus, ein Lügner und Sittenverderber zu sein. ${ }^{20}$ Trotz dieser

${ }^{18}$ Zur Personengestaltung im Nibelungenlied vgl. Ehrismann S. 212ff.; Mackensen S. 138.

${ }^{19}$ Ehrismann, S. 63; Mackensen, S. 48f. Als der historische Theoderich geboren wurde, war Attila längst tot: dieser starb 453 .

${ }^{20} \mathrm{Daß}$ freilich dieses Prinzip schon Mitte des 12. Jhs, lange vor der Blüte der höfischen Dichtung, brüchig geworden war, zeigt die Klage im Prolog der Kaiserchronik (Verse 27-40): Nu ist leider in disen zîten / ein gewoneheit wîten: / manege erdenchent in lugene / unt vuogent si zesamene / mit scophelîchen [=dichterischen] worten. / nû vurht ich vil harte / daz diu sêle 
Einengung seines kreativen Spielraums hat der Nibelungendichter die Dietrichgestalt eindrucksvoll entwickelt und damit eine bemerkenswerte Aussage gemacht. Dietrich ist zum Unparteiischen geworden, zu einem nicht voll Integrierten, der gerade deswegen als Seismograph fungieren und offen aussprechen kann, was die anderen lieber verschweigen. Das vorgezeichnete Schicksal als ellender hat der Dichter dazu benutzt, diesem Dietrich ein hohes Maß an Freiheit beizumessen. Das wird deutlich, wenn wir ihn mit anderen Protagonisten vergleichen.

Eine Funktion der männlichen Hauptpersonen im Nibelungenlied ist es, Schattierungen der Freiheit des Handelns sichtbar zu machen. Am einen Ende des Spektrums sehen wir das Kollektiv der Burgunden, samt und sonders Verstrickte, gefangen im Netz der Sippentreue und Gefolgschaft. Sie sind auch deshalb unfrei, weil auf ihnen die gemeinsame Schuld an Siegfrieds Tod lastet. Im Lauf der Handlung wird Hagen immer mehr zum Repräsentanten dieser Schar. Am anderen Ende des Spektrums steht Dietrich, der Fürst im ellende, sich frei bewegend, aber um welchen Preis? Dazwischen agieren Rüdiger von Bechelâren und Etzel. Daß die Rüdigergestalt immer wieder die Aufmerksamkeit der Forschung auf sich gezogen hat, liegt sicher mit daran, daß Rüdigers Dilemma auch in unserem Jahrhundert unverändert aktuell erscheint: Es sieht ganz so aus, als verkörpere er beispielhaft das Dilemma eines Menschen, der einerseits seinen Verpflichtungen nachzukommen hat und andererseit Gewissensfreiheit für sich beanspruchen möchte. Seine Lage ist freilich nicht ganz so 'modern'. Rüdiger befindet sich in einer auswegslosen Lage, da er beiden Opponenten Treue schuldet: Etzel und Kriemhild, weil er ihnen Eide geleistet hat, den Nibelungen, weil er durch die Verlobung Giselhers mit seiner Tochter mit ihnen versippt ist. Rüdigers Dilemma läßt sich nicht lösen und er muß kämpfend sterben, aber als Mensch leuchtet er vorher noch einen Augenblick auf. Hier sehen wir auch Hagen einen Moment in wahrer menschlicher Größe, denn er ist es ja, der die großartige Geste Rüdigers - das Geschenk des eigenen Schildes an den Feind - möglich macht.

Unter dem Gesichtspunkt der Freiheit des Handelns ist aber auch Etzel

dar umbe brinne: / iz ist ân gotes minne. / sô lêret man die luge diu chint: /die nâch uns chunftich sint, / die wellent si alsô behaben / unt wellent si iemer fur wâr sagen. / lugene unde ubermuot / ist niemen guot. E. Schröder (Hg.): Die Kaiserchronik eines Regensburger Geistlichen, Hannover 1892. 
eine wesentlich interessantere Gestalt als man üblicherweise meint. ${ }^{21}$ Etzel ist nicht nur der Landesherr und Gastgeber, der um jeden Preis versuchen muß, den Frieden zu wahren. Der Dichter hat ihn als alten Mann dargestellt, im Spannungsfeld zwischen Vergangenheit und Zukunft: zwischen der Erinnerung an seinen Zögling Hagen (eine Erinnerung, die ihm den Blick auf den realiter vor ihm stehenden grimmen man Hagen verstellt Str. 1752-1757) und dem kint Ortlieb, dessen Erziehung Etzel sich am Burgundenhof wünscht (Str. 1916-1917). Nicht die Realität des Augenblicks zählt für Etzel. Entscheidendes nimmt er nicht wahr oder läßt es nicht gelten: Hagens offen zur Schau getragene Feindseligkeit; Volkers kaltblütigen Mord an einem Hunnen während des Turniers; den von Kriemhild und Blœdelîn hinter dem Rücken des Königs begangenen Verrat. Weit wesentlicher als das alles ist dem alten König sein Traum von einer friedlichen Koexistenz mit den Burgunden. Etzel versucht - und das entspricht der Rolle des friedlichen Herrschers ausgleichend zu wirken, die Grenzen zu überwinden. Theoretisch hätte er die Gewalt dazu, unter dem Druck der Ereignisse aber wird Etzels Traum jäh zerstört: Der ehemalige Zögling Hagen erschlägt den zukünftigen Zögling Ortlieb (Str. 1960-1961). Von dem Punkt an ist Etzel dem Geschehen willenlos ausgeliefert. Die von ihm gehegte Illusion, er könne noch frei handeln, ist dahin. In der ausweglosen Lage gab es für Rüdiger noch die Chance, im Kampf ehrenhaft zu sterben. Für den altersschwachen König besteht diese Möglichkeit nicht. Gerade das dürften der Nibelungendichter und sein Publikum - ohne jede Ironie - als Etzels Tragik verstanden haben. ${ }^{22}$

Kehren wir zu Dietrich von Bern zurück. Mehrmals im Laufe des Geschehens wird er in einer Weise herausgefordert, die jeden anderen zum Schwert hätte greifen lassen. Dietrich tut das nicht. Beim Turnier durchschaut er die gefährliche Stimmung der Nibelungen und untersagt

\footnotetext{
${ }^{21}$ Diese Gedanken zur Etzelgestalt werde ich in an anderer Stelle weiter entwickeln.

${ }^{22}$ Vom Standpunkt der Geriatrie ist die Darstellung Etzels interessant. Senil ist er durchaus nicht, aber er zeigt charakteristische Symptome des Alterns. Sein Langzeitgedächtnis tritt in den Vordergrund. Die Ereignisse der fernen, wahrscheinlich verklärten Vergangenheit sowie seine friedlichen Zukunftspläne bedeuten ihm mehr als das für ihn unübersichtliche, sich allzu rasch entfaltende Tagesgeschehen.
} 
seinen Mannen die Teilnahme (Str. 1874). Dietrich ist umsichtig, läßt sich nicht provozieren, nimmt andererseits kein Blatt vor den Mund. Er ist es, der die Burgunden schon bei ihrer Ankunft am Etzelhof vor Kriemhild gewarnt hat. Als diese gewahr wird, daß ihre Gäste auf der Hut sind (sie weigern sich, die Waffen abzulegen), verliert Kriemhild einen Augenblick die Fassung. Von Dietrich wird sie mit erstaunlicher Offenheit zur Rede gestellt, zu einem Zeitpunkt, da alle anderen Protagonisten noch den höfischen Schein wahren:

"Owê mîner leide", sprach dô vrou Kriemhilt.

"war umbe wil mîn bruoder und Hagen sînen schilt

niht lâzen behalten? si sint gewarnôt.

und wesse ich, wer daz tæte, er müese kiesen den tôt."

Des antwurte ir mit zorne der fürste Dietrich:

"ich binz der hât gewarnet die edeln künege rîch

und Hagen den küenen, den Burgonden man.

nu zuo, vâlandinne, du solt michs niht geniezen lân."

Des schamte sich vil sêre daz Etzelen wîp.

si vorhte bitterlîchen den Dietriches lîp.

dô gie si von im balde, daz si niht ensprach,

wan daz si swinde blicke an ir vîande sach.

Helmut de Boor hat diese Nibelungenlied-Stelle getadelt. Dieses 'vâlandinne' sei ein Stilfehler. ${ }^{23}$ An dieser Textstelle wird aber die mit dem

${ }^{23}$ De Boor (Hg.): Das Nibelungenlied (dieser Kommentar vor ${ }^{20} 1972$ ): "1748,4 vâladinne, Teufelin. Es ist $(2371,4)$ das letzte Trutzwort Hagens gegen Kriemhild. So früh und im Munde Dietrichs verliert es sein Gewicht und ist, gleich der ganzen Zeile, ein Stilfehler des jüngsten Dichters." (S. 276). Zur Erzählweise des Dichters vgl. dagegen Ehrismann, S. 232: "Der 'Dichter' des Nibelungenliedes hat sich, den Gesetzen der Gattung gehorchend, hinter sein Werk zurückgezogen. Dieses ist mit hoher Meisterschaft erzählt, und zwar in einer Weise, die es wenig vernünftig erscheinen läßt, nicht von einem Dichter, einem Dichter zu sprechen. [...] In Verkennung der Evokationstechnik des Dichters ist man nur schwer fertiggeworden mit der Tatsache, daß dieser mit einem epischen Material spielt, das seinen Hörern zu einem Gutteil bekannt ist, das er sie zu assoziieren zwingt und das er nicht in jedem Punkt logisch entfalten möchte (vgl. Haug [in: Erste Passauer Nibelungen-Gespräche, Referat F. P. Knapp in: Universität 
mündlichen Vortrag zusammenhängende und in der heutigen Forschung überwiegend positiv kommentierte Erzählweise des Dichters sichtbar: Dieser hatte die spätere Szene noch nicht voll im Visier. ${ }^{24}$ Auf Dietrich bezogen ist diese Szene durchaus nicht mißlungen: sie ist nicht nur sinnvoll, sondern schön konzipiert: Wo die Burgunden noch höfisch schweigen, wo sogar Hagen es noch bei bissigen Anspielungen bewenden läßt, da nimmt sich Dietrich die Freiheit, offen die Wahrheit zu sagen. Kriemhild, heißt es, respektiere, ja fürchte ihn. Später verweigert ihr Dietrich und mit ihm Hildebrand - unmißverständlich jede Unterstützung gegen die Burgunden. Keinen Augenblick läßt Dietrich den Gedanken aufkommen, er könne bereit sein, Siegfrieds Tod zu rächen (Str. 1899-1902). Wieder wird klar, daß hier ein Mann spricht, der im Gegensatz zu allen anderen Freiheit zum Handeln hat und unabhängig seine Entscheidungen fällt.

Die Nachricht vom Tod seines Freundes Rüdiger und dessen Mannen trifft Dietrich zutiefst. Nach dem Ethos des Nibelungenliedes wäre eine blutige Rache durchaus gerechtfertigt. ${ }^{25}$ Dietrich verhält sich auch dies-

Passau. Nachrichten und Berichte. Nr. 43. Nov. 1985, S. 19f.]).

${ }^{24} \mathrm{Zu}$ den Widersprüchen im Nibelungenlied - die weder dem Dichter noch seinem Publikum Kopfzerbrechen bereiteten - äußert sich Mackensen ( $S$. 89f.) wie folgt: "Seine Bedeutung zu schmälern, hat man dem Dichter eine Fülle von Widersprüchen angekreidet. Warum jagt Siegfried gegen jeden höfischen Brauch mit Schwert und Schild? [...] Warum wird nicht berichtet, wo, wann, von wem die Hunnen in Etzelburg bewaffnet wurden? [...] Woher hat Dietrich den Strick, mit dem er Hagen bindet? Ist der Saal fensterlos und nur mit einem Zugang versehen, den Dankwart nach außen, Volker nach innen absperrt? [...] Besonders hat man dem Dichter seine Sorglosigkeit im Umgang mit Zahlen verübelt. Zumal mit Lebensdaten nimmt er es leicht. Hagen ist, als er zu den Hunnen zieht, etwa 80 Jahre alt. [...] Kriemhild wird mit fünfzig Jahren noch einmal Mutter [...], Brunhild trägt ihr fünfundzwanzigjähriges Kindlein auf den Armen [...]. Seine 'Widersprüche', die im einzelnen verschiedene Ursachen haben, sind in ihrer Mehrzahl Versehen, wie sie beim Diktat, der Niederschrift eines so umfangreichen Textes leicht entstanden. Nachzublättern machte bedeutende Schwierigkeiten; was beim letzten, vorletzten Mal, noch früher diktiert worden war, ließ sich nicht mehr kurzerhand nachschlagen. Fast keine der zeitgenössischen Dichtungen ist ohne solche 'Fehler'. [...] Und wer sagt uns, daß er überhaupt einen scriba zur Hilfe hatte? Wenn er, was er dichtete, selber aufschreiben mußte, vergrößerte sich die Zahl der Fehlgriffe. Möglicherweise stand er unter Zeitdruck, war alt, vielleicht krank, und wollte zu Ende kommen?"

${ }^{25}$ Sogar im Ethos des Parzival gelten sölhiu leit [...] diu herzen kumber 
mal besonnen. Er hinterfragt, überlegt, reagiert nicht mit Gewalt. Seinen Mannen untersagt er, sich provokativ zu gebärden. Daß sie es trotzdem tun und sich somit in den allgemeinen Todesstrudel hineinreißen lassen, geschieht ohne sein Wissen. Nur sein Vertrauter Hildebrand überlebt diese Katastrophe und meldet sich zurück (38. Aventiure, Str. 2235-2323). Dietrichs Klage um die jungen Gefallenen verträgt zwar noch keinenVergleich mit der berühmten Klage Willehalms um seinen im Kampf gegen die Sarazenen gefallenen Neffen Vivianz, es fällt aber auf, daß in dieser Dietrichszene schon die Sinnlosigkeit des Gemetzels und die Trauer um den Tod der Jugend anklingen, die Wolfram gut zehn Jahre später lautwerden läßt. ${ }^{26}$ In Dietrichs Klage fehlt jeder heroische Stolz auf die Gefallenen.

"Owê, lieber Wolfhart, sol ich dich hân verlorn, sô mac mich balde riuwen, daz ich ie wart geborn! Sigestap und Wolfwîn und ouch Wolfprant! wer sol mir danne helfen in der Amelunge lant?

Helpfrîch der vil küene, und ist mir der erslagen, Gêrbart und Wîchart, wie solde ich die verklagen? daz ist an mînen vreuden mir der leste tac. owê, daz vor leide niemen sterben nemac!"

Dietrich von Bern ist - zusammen mit seinem 'Schatten' Hildebrand nicht erst seit dem Tod seiner Mannen von einer Einsamkeit gezeichnet, welche den todgeweihten Burgunden fremd ist. Deren Mentalität ist eine ganz andere. Dietrich hat zwar nicht Teil an ihrem furchtbaren Ende, andererseits aber auch nicht an der wundervollen Geborgenheit, die sie im Kollektiv finden: eine Kameradschaft, deren Sinnbild Volkers Geigenspiel ist, diese Musik, von der die Burgunden sowohl in den Schlaf gesungen als auch zu blutigen Taten angefeuert werden (Str. 1833-1836). Und so

wesen (Gurnemanzlehre, 171,28-29, d.h. in der Praxis zumeist: Tötung eines Verwandten) noch als Rechtfertigung einer Blutrache. Hier im Nibelungenlied, wo die Gewaltschwelle wesentlich niedriger liegt, verliert Dietrich seinen besten Freund.

${ }^{26}$ Was bei Wolfram Willehalm (I 59,26-67,30) hervorsticht und in Dierichs Klage noch fehlt, sind die Schuldgefühle des Überlebenden, der sich für den Tod des jungen, unerfahrenen Kriegers verantwortlich fühlt. 
lassen uns die beiden Zeitgenossen - der Nibelungendichter und Wolfram von Eschenbach - angesichts der Gestalten Dietrichs von Bern und der Königin Gyburc mit der Frage zurück, ob denn die Toleranz, die Überwindung des Kampftriebes, letztlich nur Menschen möglich ist, die die Enns, diese symbolische Grenze, in sich selbst tragen, die von ihr zerrissen und von ihr in die Einsamkeit abgedrängt werden: eine Frage, die in unserer Zeit nicht weniger aktuell ist als um 1200.

Auch in der stark ins Höfische umgedeuteten Welt des Nibelungenliedes begeben sich natürlich nicht nur die Männer ins ellende. Die Frauen ziehen hier als Bräute in die Ferne, wie das in den herrschenden Klassen aller Zeiten der Fall gewesen ist. Die Brautfahrt ist seit frühester Zeit ein beliebtes Erzählmotiv, wenn auch nicht so beliebt wie das Motiv des Brautraubes. Nach den Gefühlen der Frau wird, sobald diese verheiratet ist und sich in der Fremde befindet, in den Epen jener Zeit selten gefragt. $^{27}$

Das Nibelungenlied illustriert die beiden wichtigsten Aspekte des allgemeinen Frauenschicksals: Erstens die von der Frau geforderte Unterordnung in der Ehe und zweitens das als selbstverständlich geltende Verlassen der Heimat. Brunhild - eine überhöhte, von germanischen Sagen durchwobene Gestalt - ist ein Musterbeispiel für die Frau, die gezähmt werden muß. Die übernatürlichen Kräfte der Jungfrau Brunhild, mit denen sie sich die Freier vom Leibe hält - Zeichen ihrer ursprünglich göttlichen Herkunft, aber auch Symbol der weiblichen Identität, die sie nicht preiszugeben bereit ist - werden ihr durch Betrug genommen. Als sie in der Hochzeitsnacht in einem letzten Sich-Aufbäumen gegen die Unterjochung den Ehemann an einen Nagel an der Wand hängt (Str. 630642), muß wiederum Siegfried mit seiner Tarnkappe herbei, um die Denaturierte zu bewältigen. Erst danach gelingt es ihrem Mann mit ihr zu schlafen (wir würden heute sagen: Er vergewaltigt sie), und von daweg verhält sich Brunhild wie eine 'normale' Frau (Str. 648-683). Die Zähmung dieser Widerspenstigen berichtet der Dichter mit offensichtlicher Genugtuung. ${ }^{28}$

${ }^{27}$ Eine Ausnahme bildet Enite in Hartmanns Erec. Als sie als Neuverheiratete vom Hof kritisiert wird und völlig allein dasteht, wird ihr Verlassensein einprägsam geschildert. Sie spricht von sich als mir ellendem wîbe (3030).

${ }^{28}$ Ein so heftiger Widerstand wird eher die Ausnahme gewesen sein. Viele 
Der zweite Aspekt der Brautfahrt, das Verlassen der Heimat ohne Wiederkehr und die Reise in die Fremde, wird am deutlichsten an Kriemhild sichtbar, und zwar anläßlich ihrer zweiten Eheschließung. Denn bei ihrer Fahrt mit Siegfried in die Niederlande hält sich der Dichter nicht lange auf, und von dort kehrt sie ja auch wieder nach Worms zurück, wo sie freilich, zunächst als Siegfrieds Frau und dann als seine Witwe, zum bedrohlichen Fremdkörper in der Sippe wird. Die Haltung der Brüder ihr gegenüber ist höchst ambivalent: Einerseits ist und bleibt Kriemhild die Schwester und steht nach Siegfrieds Tod wieder unter Gunthers Vormundschaft. Andererseits fürchtet man ihre Rache. Kein Zweifel, daß man Kriemhild gern los wird. Ihre Reise ins Hunnenland wird bezeichnenderweise nicht als abenteuerliche Fahrt in die Fremde dargestellt. Dahinter dürfte zweierlei stecken. Erstens die stilistische Intention. Ihre Reise dient als Kontrast zur späteren, schicksalhaftdunklen Fahrt ihrer Brüder. Zweitens aber sollte wohl die Fahrt der Frau ins Ungewisse, ihr Überqueren der Enns, prinzipiell weder als tragisches Schicksal noch als allzu aufregende aventiure dargestellt werden. Frauen waren ja aufgrund ihres Geschlechts allgemein dazu bestimmt, bei der Heirat in eine andere Sippe - ob nah oder fern - überzuwechseln. Den Gedanken, daß der Wegzug für die Braut etwas Erschreckend-Anormales sei, konnte wohl weder die Kirche noch die Gesellschaft aufkommen lassen. ${ }^{29}$ Und so wird auch Kriemhilds Reise ins Hunnenland geradezu gefeiert. Der Hof Etzels wird in diesem Erzählstadium als exemplarisch geschildert, Etzel selbst als denkbar höfisch. Daß er kein Christ ist, wird heruntergespielt: Wer weiß, heißt es während der Werbung eher beiläufig, vielleicht könne ihn Kriemhild zur Bekehrung bewegen. Aber auch wenn nicht: An seinem Hofe herrsche eine solche Toleranz, daß die Braut keinerlei Bedenken zu haben brauche (Str. 1261-1262). Von hinterlisti-

Mädchen werden die Ehe auch deshalb willig akzeptiert haben, weil sie als Angehörige des an Macht, Politik und Landbesitz orientierten Adels schon jung ein Standesdenken und ein stolzes Pflichtbewußtsein gegenüber die Sippe entwickelt hatten, das stärker war als rein individuelle Wünsche. "Ehe [hatte] vor allem mit Besitz und Reichtum [zu tun], weniger mit Selbstverwirklichung und Liebe." Opitz, S. 96ff.

${ }^{29}$ Beim erzählerischen Motiv des Brautraubes dagegen, wo das Mädchen gegen seinen Willen bzw. den des Vaters oder sonstigen Vormundes entführt wird (König Rother, Kudrun), handelt es sich nicht um weibliche Alltagsschicksale. Hier kommen aventiure und ellende voll zu ihrem Recht. 
gen, meuchelmörderischen Hunnen ist hier nicht die Rede. Dieses Motiv ist, wo es um die problemlose Eingliederung einer Braut geht, offensichtlich nicht erwünscht, ganz anders als in den späteren Szenen, wo das heimtückische und zugleich feige Verhalten der Hunnen eine bedeutende Rolle spielt. Der Nibelungendichter manipuliert also sein Publikum: Fremdenhaß wird erzählerisch gezielt eingesetzt. Kriemhilds Reise in Etzels Land gleicht mehr einer triumphalen Heimkehr als einer Fahrt ins Ungewisse. Nie wird das Fremde betont, immer die Geborgenheit, ganz im Gegensatz zur späteren Heerfahrt der Männer, die denselben Weg unter einem dunklen Schleier der Hoffnungslosigkeit zurücklegen und dann, einmal im Hunnenland angelangt, vollends im ellende sind.

In Wirklichkeit muß eine solche Brautfahrt für die meist sehr junge Frau ein traumatisches Erlebnis gewesen sein. Manche dieser Reisen sind von Chronisten, denen es bekanntlich nicht um Frauenpädagogik, sondern um Fakten ging, dokumentiert worden. So zum Beispiel die Brautfahrt der damals etwa fünfzehnjährigen portugiesischen Infantin Eleonore, die dann später die Mutter des Kaisers Maximilian werden sollte. ${ }^{30}$ Mitte des fünfzehnten Jahrhunderts fuhr sie nach Österreich, zu einem ihr unbekannten Ehemann (die Ehe mit Friedrich III., dessen Sprache und Lebensstil ihr fremd waren, war per procurationem schon geschlossen worden). Sieben Monate dauerte die Reise. Am 12. November 1451 liefen die Schiffe in Lissabon aus. Das Ziel war Talamone in Italien. Dorthin gelangte man nicht. Geplagt durch lebensbedrohliche Stürme und durch Piraten (Eleonores Schiff wäre fast gesunken) erreichte die Flotte, via Grimaud, erst Anfang Februar 1452 Livorno. Friedrich erwartete seine Braut in Florenz und wollte sie in Pisa feierlich abholen lassen. Dort traf Eleonore am 6. Februar ein, aber die Höflinge stritten eine Woche darüber, wem die Ehre gebühre, die Infantin ihrem Gemahl zuzuführen. Erst am 24. Februar trafen sich die beiden endlich in Siena. Von dort ging es weiter nach Rom, wo sie am 16. März vom Papst richtig getraut und Friedrich zum Kaiser des Heiligen Römischen Reiches gekrönt wurde. Ende März fuhr man nach Neapel. Dort verließ Friedrich - durch die

${ }^{30}$ Das folgende aus: Eberhard Holtz: "Eine Portugiesin in Österreich - Eleonore, Gemahlin Kaiser Friedrichs. III." in: Fürstinnen und Städterinnen. Frauen im Mittelalter. Hg. von Gerald Beyreuther, Barbara Pätzold, Erika Uitz, Freiburg/Basel/Wien 1993, S. 255-282. Der Chronist ist Enea Silvio Piccolomini. 
Politik genötigt - seine junge Frau. Sie fuhr weiter nach Venedig, von dort reiste sie am 2. Juni in Richtung neue Heimat ab. An der Grenze trafen die Neuver-mählten sich wieder. Österreich, berichtet der Chronist, habe das Kaiserpaar Mitte Juni mit sintflutartigen Niederschlägen empfangen, - von den politischen Wirren ganz zu schweigen. Die junge Kaiserin hatte die Enns überschritten: Die einzige Portugiesin, die ihr nach Österreich gefolgt war, starb schon zehn Monate später. Eleonore selbst wurde nur knapp dreißig Jahre alt. Portugal und ihre Verwandten sah sie nicht wieder.

Die Furcht vor dem, was jenseits der 'Enns' liegt oder vor dem, was von da drüben kommen könnte, bildet seit frühester Zeit einen Hauptgegenstand der deutschsprachigen Literatur, über mehr als tausend Jahre, nämlich vom ältesten literarischen Denkmal an, dem althochdeutschen Hildebrandslied des frühen neunten Jahrhunderts. Dort fordert ein Sohn, vaterlos aufgewachsen, einen aus der Fremde Kommenden zum Zweikampf heraus und wird - was wir aus dem Textfragment nur erraten können - von diesem in Notwehr erschlagen. Der Fremde aber (es ist der sagenumwobene Hildebrand, der dann viel später auch im Nibelungenlied eine Rolle spielt) ist sein nach Jahrzehnten aus dem ellende heimgekehrter Vater.

Die literarische Beschäftigung mit dem Verhalten an der Nahtstelle setzt sich in unseren Tagen unvermindert fort, nicht zuletzt in Österreich, das sich mehr als andere Kulturbereiche mit diesem Problem hat auseinandersetzen müssen. Ilse Aichinger machte es 1948 zum Grundmotiv ihres Romans Die größere Hoffnung. Dort sprengt das halbjüdische Kind Ellen mit ihren traumhaft-surrealen Denkmustern die erstarrten Werte der anderen. Werner Schwab ließ (in Übergewicht, unwichtig: Unform, Uraufführung 1991) seine Figuren zu Fremdenfressern werden. Elfriede Jelinek und Peter Turrini (in Stecken, Stab und Stangl, U.A. 1996, bzw. Die Schlacht um Wien, U.A. 1995) setzen sich auf der Bühne in unterschiedlicher Weise mit dem Mord an vier Roma auseinander. Die Liste ließe sich verlängern. Unermüdlich werden in diesen ansonst sehr verschiedenartigen Werken echte Grenzen definiert, falsche entlarvt, werden Wegweiser gesetzt, Brücken geschlagen und Leser wie Zuschauer nicht selten brutal aus ihrer Gleichgültigkeit herausgerissen. Es fällt auf, wie oft die Autoren selbst an der Nahtstelle stehen. Wie sie - ähnlich wie 
Gyburc und Dietrich von Bern - die Enns, diese symbolische Grenze, in sich selbst tragen und innerlich von ihr zerrissen werden. Aichinger und Jelinek sind Halbjüdinnen, - sie haben (wie es Aichinger von ihrer Persona Ellen sagt) "zwei falsche Großeltern und zwei richtige. Ein unentschiedenes Spiel." ${ }^{31}$ Für manche ist anderes entscheidend: die Identifizierung mit Randgruppen, mit sozialen, ethnischen und sprachlichen Minderheiten im eigenen Land. Heute lassen Schriftsteller und Schriftstellerinnen nicht nur einzelne fiktionale Gestalten das Entsetzen zum Ausdruck bringen, sondern sie selbst geben sich als die nicht voll Integrierten zu erkennen, die gerade deswegen als Seismographen fungieren und zur Sprache bringen, was die anderen lieber unter den Teppich kehren. Diese intensive Auseinandersetzung mit der Nahtstelle Enns ist eine literarische Tradition, deren Bedeutung weit über die Grenzen Österreichs und des deutschen Sprachraumes hinausreicht. Sie befaßt sich mit einem Grundproblem des menschlichen Daseins, über das nie genug geschrieben und diskutiert werden kann.

${ }^{31}$ Ilse Aichinger: Die größere Hoffnung, (Fischer TB) Frankfurt a.M 1974, S. 26. 